\title{
Efficacy of different irrigation solutions on the early debridement of open fracture in rats
}

\author{
QIAN CHENG ${ }^{1}$, XIAO-FEI ZHANG ${ }^{2}$, DONG-HUA DI ${ }^{1}$, GUO-YANG ZHAO ${ }^{1}$ and XUE-WEN CUI ${ }^{1}$ \\ Departments of ${ }^{1}$ Orthopedics and ${ }^{2}$ Obstetrics and Gynecology, The Affiliated Hospital of Jiangsu University, \\ Zhenjiang, Jiangsu 212000, P.R. China
}

Received April 27, 2014; Accepted November 7, 2014

DOI: 10.3892/etm.2015.2325

\begin{abstract}
Contaminating bacteria are only found on wound surfaces in the initial stages of open fractures; therefore, effective debridement is critical for bacterial infection prevention and the reduction of inflammatory reactions. Various irrigation solutions are currently being used; however, a comprehensive study on their efficacy is lacking. In the present study, a comparison of the effects of normal saline, iodophor and hydrogen peroxide as the irrigation solutions for debridement of open femur fractures in rat models was conducted. It was revealed that all three solutions were comparably effective in bacterial removal while normal saline was superior in minimizing adverse wound inflammation; therefore, the use of normal saline for routine debridement is recommended in the early-stage treatment of open fractures in the trauma clinic and in relief fieldwork.
\end{abstract}

\section{Introduction}

In the early stage of open fractures, bacteria from the open environment are attracted to the superficial layer of the wound surface and, if not removed promptly, rapidly propagate leading to wound infection. Management of wound infection often requires additional hospitalization, surgeries, and the systemic use of antibiotics, which substantial impact the patients' recovery process, quality of live, and the overall cost to the healthcare system (1). Therefore, efficient removal of bacteria from the wound surface, as the first line of treatment, is paramount to wound healing by preventing bacterial infection and minimizing inflammatory reactions (2). Wound infection is one of the oldest medical problems. In order to prevent wound infection, wound cleansing with various types of irrigation solutions has been widely performed since ancient times (3). As early as about 2000 years ago, the four principal signs of inflammation were described and the use of antiseptic

Correspondence to: Mr. Xue-Wen Cui, Department of Orthopedics, The Affiliated Hospital of Jiangsu University, 438 Jiefang Road, Zhenjiang, Jiangsu 212000, P.R. China

E-mail: cui8159@sohu.com

Key words: irrigation solution, open fractures, debridement solutions for wound cleaning. Additionally, the management of wounds had been significantly worked out (4). Among various irrigation solutions used in modern times, antiseptics such as iodophor and hydrogen peroxide have been thought to be better alternatives based on the perception that they can effectively eliminate most of the bacteria from a wound surface (5). However, it is also known that these antiseptics irritate wounded tissues leading to delayed wound healing. Therefore, it is necessary to have an irrigation solution that is both effective in bacteria elimination and favors wound healing. Different irrigation solutions are currently being used clinically for debridement (6); however, their efficacies have not yet been comprehensively investigated. In the present study, this issue was addressed by comparing the effects of normal saline, iodophor and hydrogen peroxide as irrigation solutions on the early debridement of open fractures in rat models. The present study aimed to find an effective, yet cheap and simple irrigation method, which would have a significant impact in trauma clinics, as well as in field medicine.

\section{Materials and methods}

Animals and grouping. A total of 45 healthy adult male Sprague Dawley (SD) rats, weighing between 210 and $230 \mathrm{~g}$, were provided by the Center of Laboratory Animals, Jiangsu University (Zhenjiang, China). The 45 rats were randomly divided into a normal saline group (group $\mathrm{A}, \mathrm{n}=15$ ), an iodophor group (group $\mathrm{B}, \mathrm{n}=15$ ) and a hydrogen peroxide group (group $\mathrm{C}, \mathrm{n}=15$ ). The number of rats used in each group was estimated for the significant statistical power using $\mathrm{G}^{*}$ power software (Heinrich-Heine University of Düsseldorf, Düsseldorf, Germany).

Apparatus and irrigation solutions. The gravitational impact device $(1 \mathrm{~kg}$ x $30 \mathrm{~cm})$ was self-made. Injection needles, syringes, a bench board, blades, scissors, hemostatic forceps, needle holders and a small wire retractor were purchased from local vendors. Sterile normal saline was obtained from Nanjing Xiaoying Pharmaceutical Group Co., Ltd (Nanjing, China); iodophor (containing 0.5\% iodine) was purchased from Shanghai Likang Disinfectant Hi-Tech Co., Ltd (Shanghai, China); and $0.3 \%$ hydrogen peroxide was a product of Hebei Jianning Pharmaceutical Factory (Shijiazhuang, China). 
Table I. Bacterial count before and after debridement.

\begin{tabular}{lccc}
\hline Groups & Before debridement $(\mathrm{CFU} / \mathrm{ml})$ & After debridement (CFU/ml, mean \pm SD) & Bacterial clearance $(\%)$ \\
\hline A: Saline & $>10^{5}$ & $70.00 \pm 29.03$ & 99.93 \\
B: Iodophor & $>10^{5}$ & $15.00 \pm 12.25$ & 99.98 \\
$\mathrm{C}: \mathrm{H}_{2} \mathrm{O}_{2}$ & $>10^{5}$ & $10.00 \pm 7.07$ & 99.99 \\
\hline
\end{tabular}

CFU, colony-forming unit; SD, standard deviation.

Preparation of open femur fracture models. The study design was approved by the Ethic and Animal Use Committee of Jiangsu University. Specifically, healthy adult male SD rats were anesthetized by an intraperitoneal injection of $10 \%$ chloral hydrate at a dose of $0.5 \mathrm{ml} / 100 \mathrm{~g}$ body weight, and then fixed on a bench board in a prone position. Following skin preparation, the right thigh was struck with the self-made impact device to form fractures. A $2.0-\mathrm{cm}$ incision was then made on the right thigh (Fig. 1), and the muscle tissue was separated to expose the broken ends of the fractured bone. The post-trauma rats were then uniformly placed in clean, non-sterile cages for $2 \mathrm{~h}$, allowing the open wounds to be exposed to the open air to mimic the situation in trauma patients.

Debridement. Two hours after the establishment of the models, the rats were fixed on the bench board. Prior to debridement, a sterile cotton swab was rolled twice on the wound surface and then placed in a sterile tube containing $1 \mathrm{ml}$ sterile saline for a bacterial culture. In group A, $25 \mathrm{ml}$ sterile normal saline was used to irrigate the wound surface and the broken ends of the fractured bone using a syringe. In group B, $20 \mathrm{ml}$ iodophor was used to irrigate the wound surface and the broken ends of the fractured bone via syringe. The wound was subsequently rinsed further with $5 \mathrm{ml}$ sterile normal saline. In group C, $20 \mathrm{ml}$ hydrogen peroxide was used to irrigate the wound surface and the broken ends of the fractured bone, prior to rinsing with $5 \mathrm{ml}$ sterile normal saline. Following debridement, a sterile cotton swab was rolled twice on the wound surface to collect bacteria for the culture. A $0.5-\mathrm{cm}^{2}$ sample of wound surface tissue was cut out and placed in neutral formalin for pathological examination. Finally, following the repositioning of the fractures, a sterile injection needle with a diameter of $1.2 \mathrm{~mm}$ and a length of $30 \mathrm{~mm}$ was retrogradely inserted into the femoral condyles for primary wound closure.

Postoperative treatment. Following debridement, the rat models in the three groups were separately caged and given access to food and water ad libitum. Each rat was administered an intramuscular injection of 50,000 units penicillin per day for three consecutive days, and any possible wound dehiscences, redness and swelling, exudation and other health issues were closely monitored and recorded.

Bacterial culture and count. Normal saline (10 $\mu \mathrm{l})$ taken from the tube was inoculated onto agar plates, and then incubated at $37^{\circ} \mathrm{C}$. Twenty-four hours later, the colony-forming units (CFU) from the samples collected prior and subsequent to debridement were counted and calculated for bacterial clearance. A number

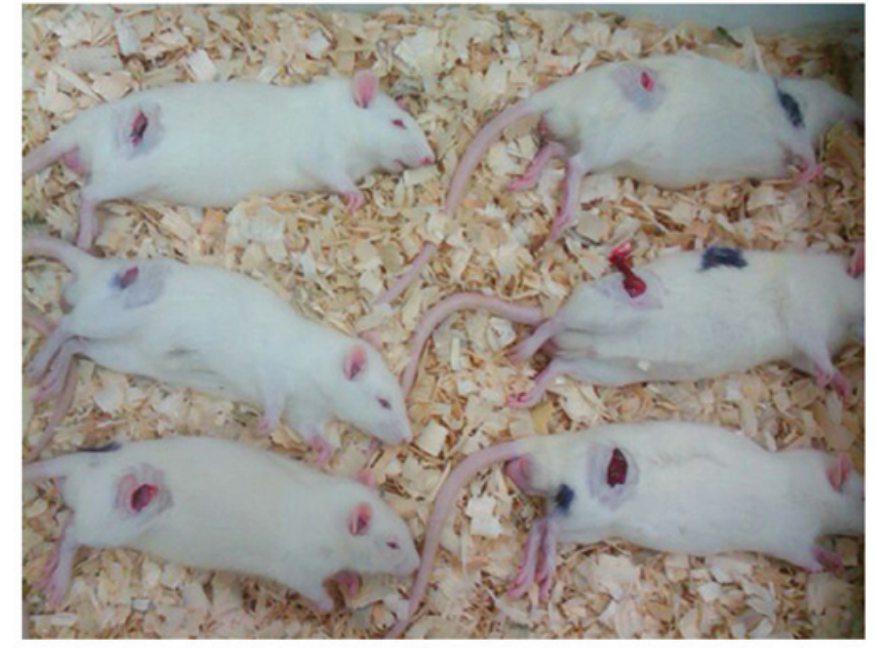

Figure 1. Image of representative rat models with open femur fractures used in this study.

of colonies were randomly selected for Gram staining, and then the bacteria were microscopically identified as Gram-positive $\left(\mathrm{G}^{+}\right)$cocci or Gram-negative $\left(\mathrm{G}^{-}\right)$bacilli bacteria.

Pathological examination. Tissue samples were fixed with neutral formalin for $24 \mathrm{~h}$, and sectioned at a thickness of $4 \mu \mathrm{m}$ followed by hematoxylin and eosin staining for the identification of inflammatory reactions.

Statistical analysis. Statistical analysis was performed with SPSS software, version 17.0 (SPSS, Inc., Chicago, IL, USA). Data are presented as the mean \pm standard deviation. $\chi^{2}$ was used for the comparisons. Differences were considered to be statistically significant at $\mathrm{P}<0.05$.

\section{Results}

General postoperative observations. A total of 45 rats were used during the study period. No animal deaths occurred and all wounds healed well. Furthermore, no wound dehiscence, redness and swelling or exudation were identified, and stitches were removed on schedule.

Pathological changes. Open femur fracture was established virtually identically in all animals (Fig. 1). Two hours after the wound establishment, debridement was performed on the animals with normal saline, iodophor or hydrogen peroxide as respective irrigation solutions. Their effects on bacte- 


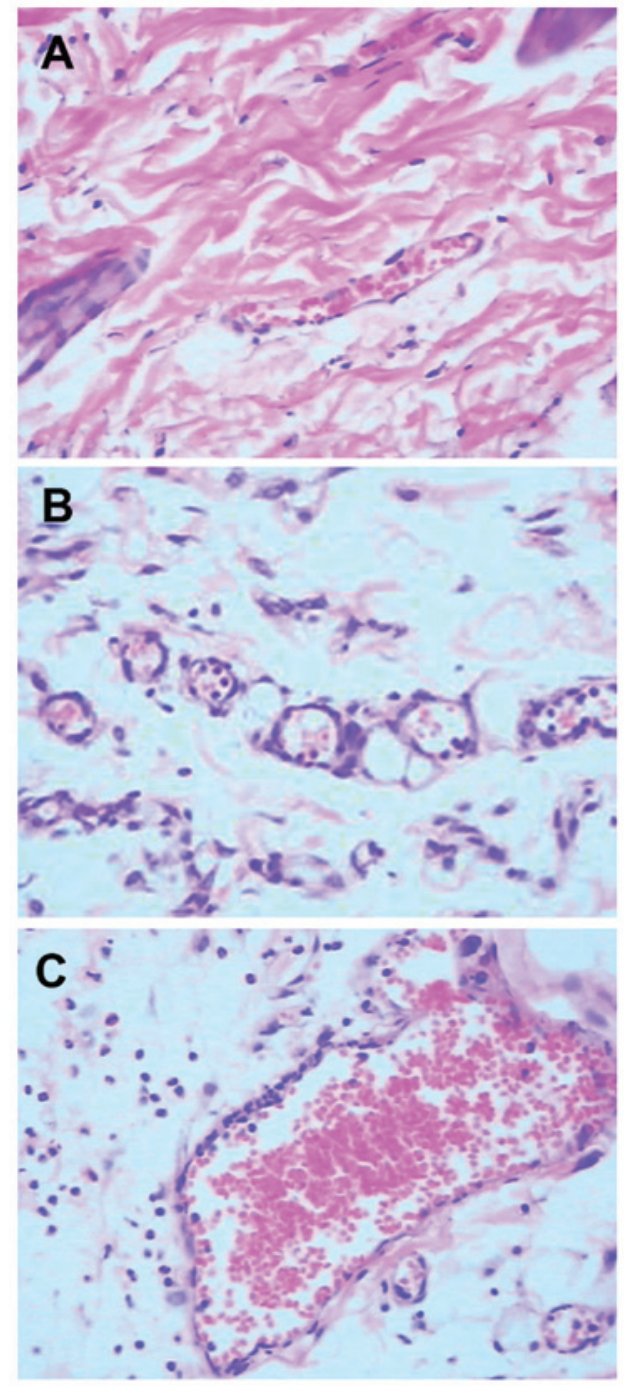

Figure 2. Pathological findings from hematoxylin and eosin stained wound tissues that had been irrigated with (A) normal saline, (B) hydrogen peroxide and $(\mathrm{C})$ iodophor. Mild vasodilation and vasocongestion were occasionally noted in (A) the normal saline-treated group, while more pronounced vasodilation, vasocongestion, slight exudation and the infiltration of inflammatory cells were clearly visible in (C) the iodophor-treated animals. Severe vasodilation, vasocongestion and exudation, along with the infiltration of inflammatory cells in interstitial tissue, were observed in (B) the hydrogen peroxide-treated group. Magnification, x40.

rial removal and wound inflammation were compared. As demonstrated by pathological changes in the tissue sections (Fig. 2), it was found that animals irrigated with normal saline exhibited the mildest inflammatory reactions, as judged by occasional mild vasodilatation and vasocongestion but not infiltration of inflammatory cells (Fig. 2A), while the animals washed with hydrogen peroxide showed evident inflammatory reactions, exhibiting severe vasodilation, vasocongestion and exudation, along with the infiltration of inflammatory cells in interstitial tissues (Fig. 2B). Finally, irrigation with iodophor produced some modest yet clearly visible vasodilation, vasocongestion, exudation and infiltration of inflammatory cells (Fig. 2C).

Bacterial clearance. Since the main purpose of irrigation is to decontaminate the wound surface from bacteria, the

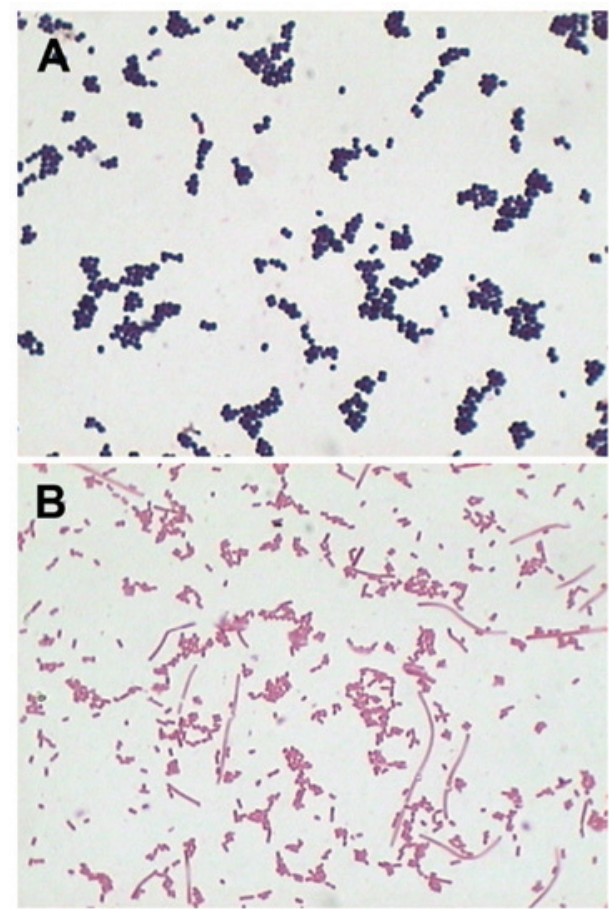

Figure 3. Representative microscopic images of cultured bacteria isolated from open wound surfaces. (A) Gram-positive cocci and (B) Gram-negative bacilli. Magnification, x100.

effects of these three solutions on bacterial removal were assessed. As shown in Table I, all three solutions were effective in removing $>99.9 \%$ of bacteria from the wound surface, as determined by CFU counts from samples collected before and after debridement. The differences in bacterial clearance were not statistically significant among the three groups $(\mathrm{P}>0.05)$ (Table I). Bacterial culture revealed that most bacteria were $\mathrm{G}^{+}$cocci and $\mathrm{G}^{-}$bacilli (Fig. 3).

\section{Discussion}

Traffic accidents are the leading traumatic events in Western countries and are becoming one of the leading types of accidents in China (7). Open bone fracture, often seen in these traffic accidents, is first treated with debridement by irrigation of a wound surface with irrigation solutions in order to remove bacteria from contaminated tissues (8-10); therefore, it is medically significant for the most effective irrigation solutions, which cause the least inflammation in the debridement treatment, to be identified.

It is clear that the wound infection rate is positively correlated with the number of bacteria retained following debridement (11). Maximal removal of bacteria from the contaminated wound surface is one of the most important measures to prevent wound infection. We believe that in the early stages of open fractures bacteria only exist on wound surfaces and have not yet had the opportunity to propagate rapidly; therefore, efficient removal of the majority of the bacteria by irrigation is critical to infection prevention. This study showed a statistically identical 99.9\% effectiveness in bacterial removal in the three groups, suggesting that those bacteria were likely to have been washed away by irrigation rather than being killed by these solutions. 
Currently, among the various irrigation solutions, normal saline, iodophor and hydrogen peroxide are all used in the clinic (12). It has been reported that the iodine in iodophor can kill bacteria, improve microcirculation at the wound surface, promote wound healing and release iodine compounds with anti-inflammatory effects (13-15); however, iodophor has toxic effects on in vitro-cultured human skin fibroblasts and immunocytes. In addition, iodine can induce and aggravate hyperthyroidism and hypothyroidism, and iodine at high concentrations can promote apoptosis, which is disadvantageous to wound healing. Hydrogen peroxide, a strong oxidant, can immediately release free oxygen upon contact with catalase in tissue. Free oxygen has anti-bacterial and disinfectant properties. Hydrogen peroxide may also cause apoptosis that is associated with neurotrophic factor, tumor necrosis factor and Fas. Catalase widely exists in tissues and can rapidly decompose hydrogen peroxide, releasing high-energy oxygen and causing chemical burn. The mechanical effects of free oxygen may allow bacterial emboli to desquamate; and then bacterial toxins, cytotoxins and gas bubbles may enter the bloodstream to activate histamine, 5-hydroxytryptamine and bradykinin, leading to hypersensitivity and even shock. A large amount of free oxygen may result in local high pressure, which allows partial bacterial toxins and gas bubbles to enter the blood through ruptures in arterioles, venules and capillaries, leading to pulmonary embolism with dyspnea, cyanosis, acute right ventricular and respiratory failure, and even mortality. It has been reported that sterile water used for irrigation in the early stages of a wound also obtains good clinical results; however, it is only suitable for superficial wounds (16). The present study indicated that normal saline resulted in the mildest inflammatory reactions among the three groups; therefore, it should be recommended as the choice of irrigation solution for early-stage open bone fracture.

In conclusion, normal saline, iodophor and hydrogen peroxide are all effective irrigation solutions in bacterial clearance following debridement, while normal saline resulted in little inflammatory reaction compared with the other two solutions. It is, therefore, conceivable to recommend using normal saline alone as the irrigation solution for the debridement of early-stage wounds in trauma clinics as well as in earthquake relief work.

\section{References}

1. Whitehouse JD, Friedman DN, Kirkland KB, Richardson WJ and Sexton DJ: The impact of surgical-site infections following orthopedic surgery at a community hospital and a university hospital: adverse quality of life, excess length of stay, and extra cost. Infect Control Hosp Epidemiol 23: 183-189, 2002.

2. Ennis WJ, Valdes W, Salzman S, Fishman D and Meneses P: Trauma and wound care. In: Morison MJ, Ovington LG and Wilkie K (eds): A Problem-Based Learning Approach. Chapter 17. Mosby Elsevier Limited, London, pp291-307, 2004.

3. Flanagan M: Wound cleansing. In: Nursing Management of Chronic Wounds. Morison M, Moffat C, Bridel-Nixon J and Bale S (eds). Mosby, London, pp221-244, 1997.

4. Honey BJ: Lizard dung and pigeons' blood. Nurs Times 80: 36-38, 1984.

5. Platt J and Bucknall RA: An experimental evaluation of antiseptic wound irrigation. J Hosp Infect 5: 181-188, 1984.

6. Gabriel A: Wound Irrigation. In Schraga, ED (eds). Medscape. http://emedicine.medscape.com/article/1895071-overview. Accessed April 2, 2014

7. Dormans JP, Fisher RC and Pill SG: Orthopaedics in the developing world: present and future concerns. J Am Acad Orthop Surg 9: 289-296, 2001.

8. Anglen JO: Wound irrigation in musculoskeletal injury. J Am Acad Orthop Surg 9: 219-226, 2001.

9. Bhandari M, Guyatt GH, Swiontkowski MF and Schemitsch EH: Treatment of open fractures of the shaft of the tibia. J Bone Joint Surg Br 83: 62-68, 2001.

10. Spencer J, Smith A and Woods D: The effect of time delay on infection in open long-bone fractures: a 5-year prospective audit from a district general hospital. Ann R Coll Surg Engl 86: 108-112, 2004.

11. McDonald WS and Nichter LS: Debridement of bacterial and particulate-contaminated wounds. Ann Plast Surg 33: 142-147, 1994.

12. Anglen JO: Comparison of soap and antibiotic solutions for irrigation of lower-limb open fracture wounds. A prospective, randomized study. J Bone Joint Surg Am 87: 1415-1422, 2005.

13. Mueller S, Vogt PM, Steinau HU, Leuner C, Hopp M, Bosse B, Fleischer W and Reimer K: Repithel: removing the barriers to wound healing. Dermatology 212 Suppl 1: 77-81, 2006.

14. Beukelman CJ, van den Berg AJ, Hoekstra MJ, Uhl R, Reimer K and Mueller S: Anti-inflammatory properties of a liposomal hydrogel with povidone-iodine (Repithel) for wound healing in vitro. Burns 34: 845-855, 2008

15. Langer S, Botteck NM, Bosse B, Reimer K, Vogt PM, Steinau HU and Mueller S: Effect of polyvinylpyrrolidone-iodine liposomal hydrogel on wound microcirculation in SKH1-hr hairless mice. Eur Surg Res 38: 27-34, 2006.

16. Trevillion N: Cleaning wounds with saline or tap water. Emerg Nurse 16: 24-26, 2008. 